\title{
Features of Fundamental Plane for Early-Type Galaxies by Clausius' Virial theory
}

\author{
L. Secco \\ Department of Astronomy, University of Padova, Padova, ITALY $\dagger$
}

\begin{abstract}
The theory of the Fundamental Plane (FP) proposed by Secco (2005) is based on the existence of a maximum in the Clausius' Virial (CV) potential energy of a stellar component when it is completely embedded inside a dark matter (DM) halo. At the first order approximation the theory was developed by modeling the two-components with two power-law density profiles and it produces some expectations in fairly good agreement with the observations. We add other predictions of the theory at the same level of approximation about the Zone of Exclusion (ZOE) in $\mathrm{k}$-space and its possible relationship with cosmological scenario. Some of the consequences of the thermodynamical properties of CV maximum are also taken into account.
\end{abstract}

\section{Results}

The virial equilibrium is described by tensor virial equations extended to two subcomponents (Brosche et al., 1983; Caimmi \& Secco, 1992). The interaction terms are analitycally performed along the sequences of $\mathrm{CV}$ as function of the ratio baryonic to halo virial semi-axis for different values of the dark to bright mass ratio $m$. Among the infinity virial configurations a baryonic stellar component of a given mass may assume inside a dark matter halo of fixed mass and shape, a special configuration exists if $m$ is greater than a given threshold. That seems strictly connected with the ZOE existence (Burstein et al., 1997; D'Onofrio et al., 2006). The corresponding slope of the limit line results depending on the cosmology. At this approximation level the thermodynamics turns out to be very useful in order to indicate why the slope of the dark matter halo profile has to be so shallow in order to obtain a CV maximum with a strong "attractivity". Even if the theory needs to an higher level of approximation in order to be suitable to more realistic ETG models, the results are relevant in order to understand how the $\mathrm{CV}$ theory may be the engine for some main features of galaxy FP.

\section{References}

Brosche, P., Caimmi, R., \& Secco, L. 1983, A\&A, 125,338.

Burstein, D., Bender, R., Faber, S.M., \& Nolthenius, R. 1997, AJ, 114,1365.

Caimmi, R. \& Secco, L. 1992, ApJ 395, 119.

D’Onofrio, M., Valentinuzzi, T., Secco, L., Caimmi, R., \& Bindoni, D. 2006, New Astron. Revs $50,447$.

Secco, L. 2005, New Astron. 10, 439.

$\dagger$ Acknowledgment:Particularly to the friend R.Caimmi, to M.D'Onofrio, T.Valentinuzzi, D.Bindoni for the useful discussions and help and to Fondazione Cassa di Risparmio di Padova e Rovigo for financial support. 